\title{
Novel Energy Model To Analyze The Effect Of MAC And Network Parameters On Asynchronous IEEE 802.15.4 Multi-hop Wireless Networks Lifetime
}

\author{
Raja Vara Prasad Y and Rajalakshmi Pachamuthu \\ Department of Electrical Engineering \\ Indian Institute of Technology Hyderabad, India \\ Email: [ee10p004,raji]@iith.ac.in
}

\begin{abstract}
Design of energy efficient wireless networks is the primary research goal for evolving billion device applications like Internet of Things (IoT), smart grids and Cyber Physical Systems (CPS). Energy efficient models can reduce megawatts of power through out globe thereby reducing carbon foot print by improving overall network lifetime of large scale networks. Recent advances in physical layer have optimized energy consumption of IEEE 802.15.4 Physical layer, but energy efficiency of MAC and network layers is also essential to realise Green wireless networks. Though anycast multi-hop communication is initiated by many recent researchers by obtaining reliability, delay and energy expressions, still there is no model that captures relay nodes state wise behaviour effectively. In this paper a new energy model with 3-dimensional Markov MAC model with generalized anycast routing and state behaviour is proposed and developed for asynchronous wireless ad hoc networks. Proposed state behaviour of node model has consideration of MAC and network parameters like minimum backoff exponent, maximum backoff stages and retries along with network parameters like packet length, wake up rate, Sleep, Idle-Listen, Active-Tx and Carrier Sense Multiple Access with Collision Avoidance (CSMA/CA) states. Results show that total energy of derived model depends on many network and MAC parameters. Affect of most of the considered parameters are analysed with simulation results. Total energy of the multi-hop network reduced to $25 \%$ with variation in minimum backoff exponent and increased by $45 \%$ with increase in packet length. It is observed that derived analytical energy model better fits with most of the parameters that affects energy.
\end{abstract}

Keywords-Clustered multi-hop network, Anycast routing, Markov chain, Wake up rates, End-to-end delay, Reliability.

\section{INTRODUCTION}

The IEEE 802.15.4 standard in [1] has become default protocol for low data rate and low power wireless sensor network (WSN) applications in industry, control, home automation, health care, and smart grids [2] and [3] thereby enabling Internet of Things (IoT). IoT has 50 billion devices connected by the year 2020 according to the report in [4]. When such a large number of devices exists, one would like to design more energy efficient wireless network thereby transforming into Green wireless networks. Wireless networks without clock synchronization (asynchronous wireless network) can be opted for dense IoT networks to avoid complexity and central infrastructure. This paper focuses on developing an energy model for green wireless networks based on 3-dimensional Markov chain with anycast routing. In anycast routing relay nodes broadcast beacons to its nearest neighbours when it is ready to receive data and waits for a beacon from its nearest nodes when it wants to forward data.

Busy channel probabilities in Clear Channel Assessment (CCA) slots, $\alpha$ (CCA1), $\beta$ (CCA2) and channel sensing probability $(\tau)$ are derived in [5] with minimum backoff exponent $\left(m_{0}\right)$ and maximum backoff stages $(m)$ as 2 dimensions of Markov chain for both slotted and unslotted scenarios. Reliability and delay constrained packet delivery is discussed in [6]. It is known that IEEE 802.15.4 may have poor performance in terms of power consumption, reliability and delay [7], unless the medium access control (MAC) parameters are properly selected. Energy is the utmost priority in wireless sensor networks (WSN) because of tiny battery powered nodes. Most of the proposals/protocols [6], [7] and [8] considered energy as the primary objective constrained on reliability and delay. All works discussed above from literature don't have a generalized solution for large multi-hop networks. Analytical models in [5] and [6] are targeted for single hop communication. [8] proposed a novel multi-hop model with anycast routing. Though model in [8] has Sleep, Idle, Active and CSMA/CA states, analytical model could not capture state wise behaviour of relay node. Model in [8] considered only cumulative wake up rate and number of hops as major variables leaving behind the effect of most MAC and network parameters on performance metrics like reliability, delay and energy. In order to enhance the network lifetime and improve the quality of the service experienced by the applications, analytical energy model of IEEE 802.15.4 protocol with most of the MAC and network parameters is essential.

This paper focuses on energy model of IEEE 802.15.4 for clustered multi-hop networks with anycast routing and different states like Sleep, Idle-Listen, Active-Tx and CSMA/CA. 
State wise behaviour of relay and sensor nodes in cluster optimizes energy usage using optimal Sleep and Idle-Listen schedules instead of continuous listening mode. Proposed energy model has possible network parameters like minimum backoff exponent $\left(m_{0}\right)$, maximum backoff stages $(m)$, retries $(n)$, packet arrival rate $(\lambda)$, wake up rate per node $(\mu)$, packet length $\left(L_{p}\right)$ and number of nodes $(N)$ along with sleep time $\left(L_{s}\right)$, active time $\left(L_{a}\right)$ and idle time $\left(L_{i}\right)$, etc.

The rest of this paper is as follows. In section 2, we summarize existing work on analytical energy model of IEEE 802.15.4. Section 3 presents the system model based on a generalized Markov chain model of CSMA/CA with anycast clustered multi-hop communication. Section 4 presents state wise energy models and total energy model of the proposed system. The analytical model results with variation in MAC and network parameters is discussed in section 5. Finally, section 6 concludes the paper.

\section{RELATED WORK}

The analysis of IEEE 802.15.4 WSN's packet delay, throughput, and power consumption has been the focus of several simulation based studies in [9] and [10]. Energy consumed per unit data transmission and reception is discussed in [11]. This model computes energy of a single hop topology without considering many MAC and network parameters. A Markov based model analysing throughput and energy is discussed in [11]. [12] discusses a star topology model with Markov chain considering contention access period of IEEE 802.15.4. Effect of packet arrival rate on throughput and energy is well analysed in [12], but lacks detailed state wise behaviour of relay node.

Some of the proposed protocols focuses on wireless system which can guarantee reliable and timely data [6] for the star networked systems. Energy consumption model in [6] considers only transmit, receive and idle energies of star network. This model well analysed the star network energy with variation in MAC parameters. Analytical model proposed in [8] is based on clustered multi-hop network with wake up rates. Even though Markov model in [8] has detailed analysis on reliability, delay and energy parameters, Markov model has been proposed without retries and node state behaviour. Model in [8] also lacks energy analysis with different MAC parameters and network parameters. In this paper a 3-dimensional Markov chain embdedding state wise energy model with possible parameters like minimum backoff exponent $\left(m_{0}\right)$, maximum backoff stages $(m)$, packet length $\left(L_{p}\right)$, active and idle times is proposed. Effect of the above parameters are analysed with analytical model developed. To the best of our knowledge, there is no such model for multi-hop networks which can capture most possible MAC, network and state wise parameters unlike the model presented in this paper.

\section{SYSTEM MODEL}

System proposed is a wireless Cyber Physical System (CPS) with a centralized sink/gateway with distributed sensors and relay nodes as shown in the Fig. 1 . An example scenario with 4

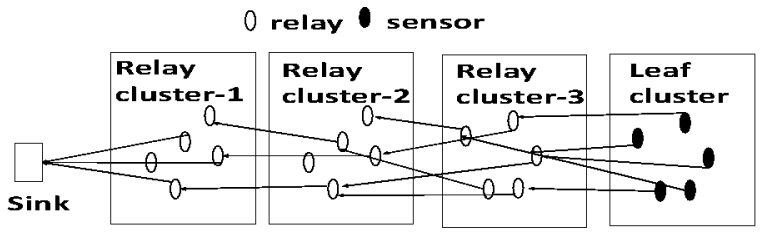

Fig. 1: Proposed system model

clusters is considered for modeling and analysis in this paper. Sensor data in the leaf cluster is to be routed to gateway via multiple hops through relay clusters. Random routing mechanism namely anycast routing is used to route data from source (sensor) to destination (sink). In anycast routing relay nodes broadcast beacons to its nearest neighbours when it is ready to receive data and waits for a beacon from its nearest nodes when it wants to forward data. Relay nodes are modeled to follow poisson random process of wake up instances instead of listening all the time. For example if a sensor node in leaf cluster need to send data to sink, First it will wait for a beacon from any of its nearest relay node in forwarding cluster (cluster-3). If sensor node succeeded in receiving beacon it transmits data using CSMA/CA mechanism. If any relay node in cluster-3 successfully receives packet from sensor node in leaf cluster, then relay node repeats same procedure as sensor node to transmit the received data to cluster- 2 node. This anycast routing is repeated by intermediate relay nodes till the data reaches sink node. Brief description for 4 different states of a relay node namely Sleep, Idle-Listen, Active-Tx and CSMA/CA is given below shown in Fig. 2. Detailed description of node model is given along with mathematical formulation in following sections. Before presenting the energy model brief description of the states are given as below.

- Sleep state: Relay node sleep until assigned wake up time.

- Idle listen state: Relay node wakes up and forwards a beacon to it's predecessor cluster (leaf cluster is predecessor for cluster-3) nodes indicating that it is ready to accept data. Idle time is decided upon the maximum amount of time to receive a data packet.

- Active-TX state: Relay after receiving a packet wait's for active timeout to receive a beacon from neighbor forward cluster (cluster-2 is forward to cluster-3). In Active-Tx state node waiting time depends on poisson process of relay node wake up instants.

- CSMA/CA state: Relay node follows CSMA/CA algorithm shown in Fig. 3. The Markov model proposed in this paper is explained below.

Assumptions made in developing the model are given as follows:

- Relay nodes are uniformly distributed and static.

- Proposed system model assumes congestion due to Acknowledgements is very negligible.

- System also assumes dual channel operation at every relay node. Relay node in each cluster receives on one channel and transmits on other channel to reduce inter- 


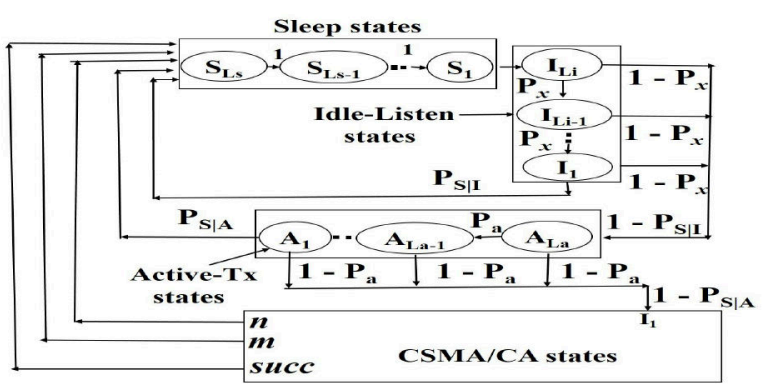

Fig. 2: 3-dimensional Markov model with state wise behaviour

ference from same cluster nodes and co located nodes.

- All nodes are assumed having accurate CCA and errors due to reduced sensing is negligible.

- Beacons are transmitted on different channel other than data channels.

- Relay nodes are assumed to follow poisson wake up instants.

- Perfect channel conditions are assumed. Packet failure due to channel conditions like fading and interference from other $2.45 \mathrm{GHz}$ user is assumed negligible.

A three dimensional Markov chain based mathematical models with $s(t), c(t)$ and $r(t)$ as three dimensions, where $s(t)$ is stochastic processes representing the backoff stage, $\mathrm{c}(\mathrm{t})$ is the state of the backoff counter and $\mathbf{r}(\mathrm{t})$ is state of retransmission counter shown in Fig. 3. All the above models focused on deriving reliability, delay and energy without consideration of Sleep, Idle-Listen and Active-Tx state behaviour. Above mentioned states strongly affects the network life time if Sleep, Idle-Listen and Active-Tx states are not scheduled optimally. Though above Markov based studies [6], [7] and [14] has done serious investigation in this area, but we feel that main objective function energy in above studies lacks state wise behaviour, MAC and network parameters. This paper focus on deriving an efficient energy model for clustered anycast multi-hop network using state wise behaviour of relay nodes. New Markov chain model along with states is proposed as shown in the Fig. 2.

State wise probabilities are essential to arrive at proposed energy model in this paper. In Eq. 1, $P_{x}$ gives the transition probability of a relay node to move into next Idle-Listen slot from the current, when there is no packet arrival at a given slot with an average of $\lambda$ poisson arrivals and $P_{a}$ indicates the transition probability of a relay node to move into next ActiveTx slot from the current, when there is no beacon arrival at a given slot with an average of $\lambda_{a}$ poisson arrivals shown in Eq. 4. In Eq. 2 and 3, $P_{S \mid I}$ and $P_{S \mid A}$ are the transition probabilities of a node from Idle-Listen and Active-Tx states to first slot of Sleep state respectively. $L_{a}$ and $L_{i}$ are length of active and idle slots respectively. $\mu$ indicate wake up rate per node and $\mathrm{N}$ is the number of nodes per cluster. $\lambda$ and $\lambda_{a}$ are packet arrival rate and beacon arrival rate respectively from Eq. 1 and 4 . In Eq. 6, $P_{S \mid C S M A}$ is the transition probability of a node from CSMA/CA state to first slot of Sleep state.

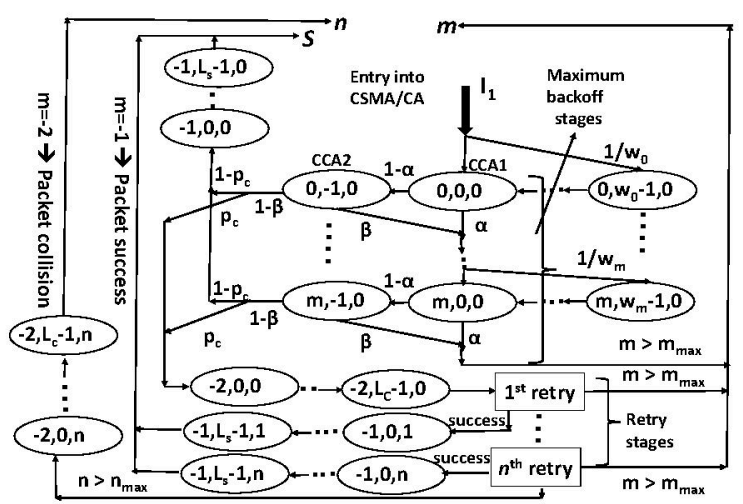

Fig. 3: 3D Markov chain model of IEEE 802.15.4 CSMA/CA

$$
\begin{gathered}
P_{x}=\exp (-\lambda) \quad, \quad P_{a}=\exp \left(-\lambda_{a}\right) \\
P_{S \mid I}=P_{S_{L_{s}} \mid I_{1}}=P_{x}^{L_{i}} \\
P_{S \mid A}=P_{S_{L_{s}} \mid A_{1}}=P_{a}^{L_{a}} \\
\lambda_{a}=\mu * N \\
P_{S \mid C S M A}=P_{S_{L_{s}} \mid C S M A_{m}}+P_{S_{L_{s}} \mid C S M A_{n}} \\
+P_{S_{L_{s}} \mid C S M A_{s u c c}}=1 \\
P_{S_{0}}=P_{S_{0}} P_{x}^{L_{i}}+P_{S_{0}}\left(1-P_{x}^{L_{i}}\right) P_{a}^{L_{a}}+P_{S \mid C S M A} b_{0,0,0}
\end{gathered}
$$

In Eq. 5, $P_{S_{L_{s}} \mid C S M A_{m}}$ and $P_{S_{L_{s}}} \mid C S M A_{n}$ are the probabilities to enter into first slot of Sleep state when packet is discarded in CSMA/CA after exceeding maximum $m$ and $n$ respectively. $P_{S_{L_{s}} \mid C S M A_{\text {succ }}}$ is the probability of the node to enter first slot of Sleep state after the node successfully forwards packet with an ACK reception. Eq. 6 can be simplified to arrive at probability $P_{S_{0}}$ of a node to stay in first sleep slot at any given time slot in terms of $b_{0,0,0}$, where $b_{0,0,0}$ is the probability of a node to stay in the first CCA1 slot of the CSMA/CA model. Using normalization property of Markov chains in Eq. 7, we derive an expression for $b_{0,0,0}$.

$$
P_{S}+P_{I}+P_{A}+P_{C}=1
$$

In Eq. $7 P_{S}, P_{I}, P_{A}$ and $P_{C}$ are probabilities for a node to reside in Sleep, Idle-Listen, Active-Tx and CSMA/CA states respectively at a given time slot. $\alpha, \beta$ and $\tau$, where $\alpha$ is the probability of channel busy in CCA 1 state, $\beta$ is the probability of channel busy in CCA2 state and $\tau$ is the channel sensing probability should be rederived with all possible state probabilities from Eq. 1 to 7 along with CSMA/CA probabilities. One can refer [5] and [14] to arrive at detailed derivations of new $\alpha, \beta, \tau$ and $b_{0,0,0}$. New $\alpha, \beta$ and $\tau$ expressions obtained from $b_{0,0,0}$ are highly nonlinear and don't have a closed form solution. Numerical methods (Newton-Raphson) are used to arrive at $\alpha, \beta, \tau$ and $b_{0,0,0}$. Eq. 8 gives the probability of being channel busy in either CCA1 or CCA2.

$$
x=\alpha+(1-\alpha) * \beta
$$

Eq. 9 gives the probability of node taking $n$ retries when it fails to transmit packet due to collision. All the above mentioned 
probabilities like $\alpha, \beta, \tau$ and $b_{0,0,0}$ are used in the energy model expressions of the next section.

$$
\widehat{y}=\left(1-(1-\tau)^{\mathrm{N}-1}\right) *\left(1-x^{m+1}\right)
$$

\section{ENERGY MODEL}

Energy consumption for the proposed anycast multi-hop network is summation of individual state energies of all nodes shown in Eq. 10. State energies should be calculated to get total energy consumption as below. Idle, Active-Tx, CSMA/CA, wake up, beacon, transmit and receive state energies are to be formulated in order to arrive at total energy. Total energy can be calculated after deriving individual state energies for endto-end anycast communication. $E_{i}, E_{a}, E_{\mathrm{CCA}}, E_{w}, E_{t}$ and $E_{r}$ are energy consumption in idle, active, CSMA/CA sensing, wake up, transmit and receive states respectively.

$$
E_{\text {Total }}=\left(E_{i}+E_{a}+E_{w}\right)+E_{\mathrm{CCA}}+E_{t}+E_{r}
$$

1) Idle listen energy: A relay node in cluster waits for a packet from predecessor cluster. Energy consumed during this state depends on poisson process of packet arrivals. Probability a node to reside in idle state is calculated as in Eq. 11 to get idle energy of a relay node. Since arrival of packets are poisson process, the probability of a relay node in idle listen state is formulated as below.

$$
P_{\text {idle }}=\frac{\exp (-\lambda) *(1-\exp (-\lambda))}{(1-\exp (-\lambda * L i))}
$$

Average number of idle slots that a node waits before a packet arrives is given in Eq. 12.

$$
L I_{\text {idle }}=\sum_{i=0}^{\mathrm{Li}} \frac{\exp (-\lambda) *\left(1-\exp \left(-\lambda_{i}\right)\right)}{\left(1-\exp \left(-\lambda_{i} * L i\right)\right)}
$$

Finally energy in Idle-Listen state is given in Eq. 13.

$$
E_{i}=T * \mu * N * h * L I_{\text {idle }} * P_{i} * s b
$$

Where $h$ is the number of hops, $s b$ is the unit backoff period, $\mathrm{T}$ is the time of consideration and $P_{i}$ is the average idle power.

2) Active energy: A relay node which successfully received a packet from predecessor cluster has to transmit received packet to next nearest cluster nodes. Relay node in ActiveTx state waits for a beacon from forwarding cluster nodes (cluster-2 is forwarding cluster to cluster-3) to start CSMA/CA procedure. Probability of a node in Active-Tx state is formulated to calculate active energy of a relay node as shown in Eq. 14.

$$
P_{\text {active }}=\frac{\exp \left(-\lambda_{a}\right) *\left(1-\exp \left(-\lambda_{a}\right)\right)}{\left(1-\exp \left(-\lambda_{a} * L a\right)\right)}
$$

Average number of active slots that a node waits before a beacon arrives is given by Eq. 15

$$
L A_{\text {active }}=\sum_{i=0}^{\mathrm{La}} \frac{\exp \left(-\lambda_{a}\right) *\left(1-\exp \left(-\lambda_{a}\right)\right)}{\left(1-\exp \left(-\lambda_{a} * L a\right)\right)}
$$

Finally energy in Active-Tx state is given in Eq. 16.

$$
E_{a}=T * \mu * N * h * L A_{\text {active }} * P_{a} * s b
$$

Where $P_{a}$ is the average active power.

3) CSMA/CA channel sensing energy: Relay node in CSMA/CA state waits for a clear channel assessment. Energy for channel sensing is obtained from CCA1 and CCA2 state probabilities $\left(b_{i, 0, j}\right.$ and $\left.b_{i,-1, j}\right)$ of 3-dimensional Markov chain in Fig. 3 shown in Eq. 17. Energy during backoff mechanism is considered zero as relay node is assumed in sleep state instead of idle listen state. Energy during channel sensing is given in Eq. 18.

$$
\begin{gathered}
E_{\mathrm{CCA}}=P_{\mathrm{sc}} * \sum_{i=0}^{m} \sum_{j=0}^{n}\left(b_{\mathrm{i}, 0, \mathrm{j}}+b_{\mathrm{i},-1, \mathrm{j}}\right) \\
E_{\mathrm{CCA}}=T * \mu * N * h * P_{\mathrm{sc}} *(2-\alpha) * \tau
\end{gathered}
$$

where $b_{\mathrm{i}, 0, \mathrm{j}}, b_{\mathrm{i}, 1, \mathrm{j}}$ are Clear Channel Assessment (CCA) states CCA1 and CCA2 respectively and $P_{\mathrm{sc}}$ is average power consumption in channel sensing states.

4) Transmit and Receive energies: Transmit and receive energies are also derived from 3-dimensional Markov model of CSMA/CA. Probability of a relay node in transmit state when it is performing CSMA/CA algorithm is used to arrive at transmit and receive energy calculations as given in Eq. 19 and 20 .

$$
E_{t}+E_{r}=P_{t} * \sum_{i=0}^{m} \sum_{k=0}^{\mathrm{Lp}-1} \sum_{j=0}^{n} b_{\mathrm{i}, \mathrm{k}, \mathrm{j}}+\sum_{j=0}^{n} \sum_{k=L p+1}^{\mathrm{Lp}+\text { Lack }+1}\left(P_{r} * b_{-1, \mathrm{k}, \mathrm{j}}\right)
$$

$$
\begin{array}{r}
E_{t}+E_{r}=T * \mu * N * h *(1-x) * \tau * \\
\left(P_{t} * L p+\operatorname{Lack} *\left(P_{r} *\left(1-P_{c}\right)\right)\right.
\end{array}
$$

where, $P_{c}$ is collision probability, $P_{t}$ and $P_{r}$ are average transmit and receive powers taken from data sheet of Tmote sky [15]. Lack is length of ACK slots.

5) Awake and beacon transmit energy: when ever a relay node wake up it involves wake up energy due to hardware circuitry. This energy depend on the wake up rate formulated in Eq. 21. Anycast multi-hop model is used to realize multihop communication. Beacon is broadcasted whenever a node is awake. Beacon transmit energy depends on wake up rate per node. Transmit power for beacon is assumed same as transmit power for data packet which is given in Eq. 21. $P_{h}$ is energy due to hardware circuitry.

$$
E_{w}=T * \mu * N * h *\left(P_{h}+P_{t}\right)
$$

\section{ANALYTICAL RESULTS}

Proposed analytical energy model in this paper is analysed with Matlab simulations. Energy model derived has several components which in turn depends on MAC parameter like $\operatorname{minBE}\left(m_{0}\right)$ and maxbackoff stages $(m)$. Model also shows 

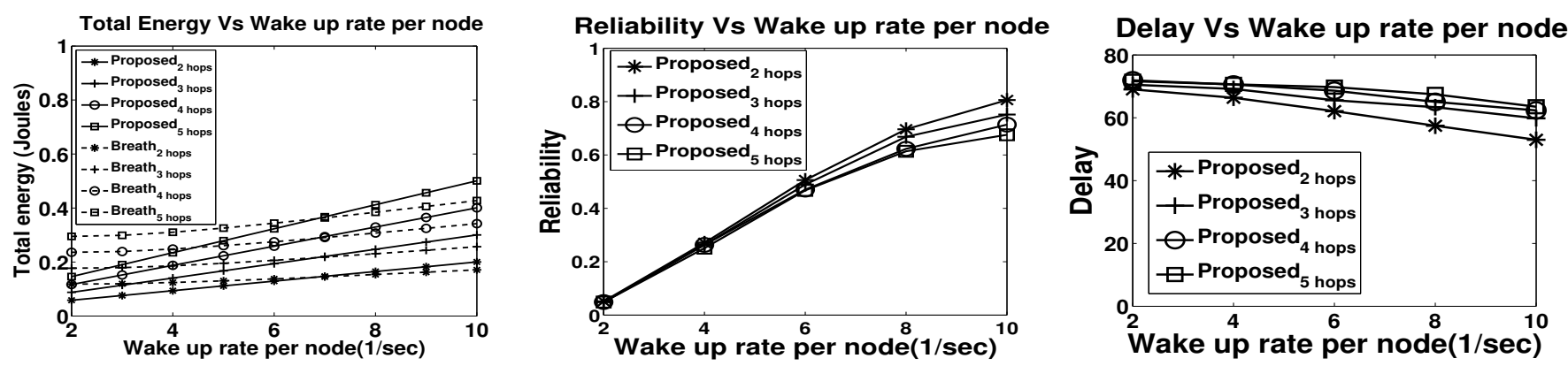

Fig. 4: a) Total energy Vs $\mu$; b) Reliability Vs $\mu$ c) Delay Vs $\mu$; Simulation parameters for all 3 plots are $m_{0}=3, \mathrm{~m}=4, \lambda$ $=5$ packets/sec, $n=1, \mu_{w}=[210], L_{a}=100, L_{p}=10, L_{i}=100, L_{s}=100$.

variation with network parameters like wake up rate $(\mu)$, packet length $\left(L_{p}\right)$ and number of clusters $(h)$ etc. A scenario similar to Fig. 1 with 4 clusters (including leaf sensor cluster) is taken for analytical simulation. Each cluster is assumed of 5 nodes. Leaf sensor node generates $\lambda$ packets in a second. Total energy of the proposed model with variation in wake up rate is compared with model in [8].

\section{A. Effect of wake up rate $(\mu)$ on energy}

Proposed energy model has wake up rate per node which follows a poisson process. Effect of this wake up rate on total energy is analyzed in this paper shown in the Fig. 4(a). Increase in wake up rate resulted in increase in energy due to beacon transmission and additional hardware circuit consumption shown in Fig. 4(a). Fig. 4(a) plots a comparison of total energy of our proposed model and model in [8] in Joules versus wake up rate per node per second. Each curve represents variation in total energy with $\mu$. Total energy of the proposed model is increasing linearly in the range of [0.05 0.2] for 2-hop scenario. Linear increase in total energy is due to linear relation of $E_{i}, E_{a}, E_{C C A}, E_{w}$ and $E_{t}$ with $\mu$. Total energy is increasing with number of hops as number of nodes should wake up to forward data increases. Total energy of the proposed model is less than Breath model up to 7 wake ups shown in the Fig. 4(a). Total energy is observed less at lower wake up rates compared to Breath which is an essential quality of the model. Total energy of the proposed model is 30\% less than Breath at lower wake up rates. Total energy of the proposed model for 5-hops scenario is much less than Breath shown in the Fig. 4(a). Above result proves that proposed model has greater advantage for larger network at lower wake up rates compared to Breath. Results in Figs. 5, 6 and 7 are not compared with Breath model as the parameters of interest like $m_{0}, m$ and $L_{p}$ are fixed parameters unlike in the proposed model.

Even though our model is targeted for energy saving but reliability and delay performance metrics of the proposed model are not compromised much as shown in the Figs. 4(b) and 4(c). Reliability and delay models of the proposed model in this paper is not included due to space constraints. Reliability Vs wake up rate of the proposed model is plotted in Fig. 4(b) for 2, 3, 4 and 5 hops. Reliability increases in the range of [0.1 0.8$]$ with only $25 \%$ increase in energy for 2-hops scenario. Increase in reliability is due to increase in average available relay nodes to forward data. Similarly delay Vs wake up rate is plotted in the Fig. 4(c). Delay is decreasing in the range of [70 60] milliseconds for 2-hop network. Decrease in the delay is due to decrease in average waiting time to forward a packet in Active-Tx state. It is clear from Figs. 4(a), 4(b) and $4(c)$ that reliability and delay are not degrading significantly with decrease in energy consumption.

\section{B. Effect of minimum backoff exponent $\left(m_{0}\right)$ on energy}

Effect of $m_{0}$ is analysed on total energy. Fig 5 plots total energy versus variation in $m_{0}$ where each curve in Fig. 5 is variation in number of hops. Total energy is decreasing with increase in $m_{0}$ as shown in Fig. 5. Decrease in energy is due to increase in backoff windows of relay nodes when there is congestion in the network. Energy spent in backoff is considered as sleep energy in this model which is a valid assumption. Total energy considerably reduced which is in the range of [0.58 0.15] Joules for 5 hops. Total energy of the multi-hop network reduced to $25 \%$ with variation in minimum backoff exponent for 5 hops.

\section{Effect of maximum backoff stages $(m)$ on energy}

Effect of $m$ is analysed on total energy. Fig. 6 plots total energy versus variation in $m$ where each curve in Fig. 6 is variation in number of hops from 2 to 5 . Total energy is decreasing marginally with increase in $m$ shown in Fig. 6 . Decrease in energy is due to increase in backoff stages of relay nodes when there is congestion in the network. Increase in number of backoff stages will force relay nodes to remain in backoff sleep mode. Energy spent in backoff stage is considered as sleep energy in this model which is a valid assumption. Marginal decrease in total energy with variation in $m$ is due to lower probability that a node takes a next backoff stage every time. Total energy increases with increase in number of hops due to increase in number of nodes in the network. Total energy is observed in the range of [0.56 0.55] Joules with variation in $m$ for 5 hops.

\section{Effect of packet length $\left(L_{p}\right)$ on energy}

Effect of $L_{p}$ is analysed on total energy. Fig. 7 plots total energy versus variation in packet length $\left(L_{p}\right)$ in slots where 


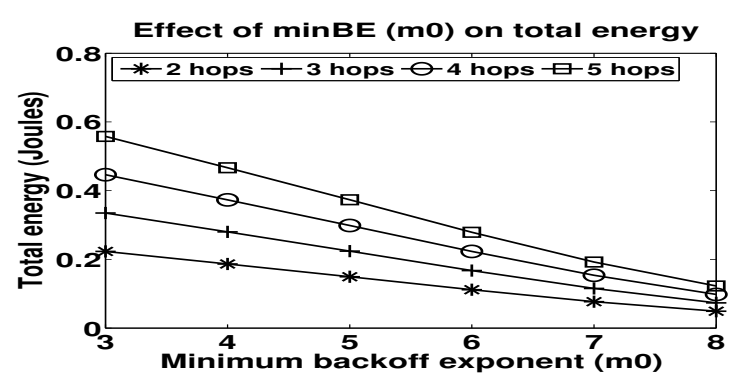

Fig. 5: Effect of $m_{0}$ on Total energy

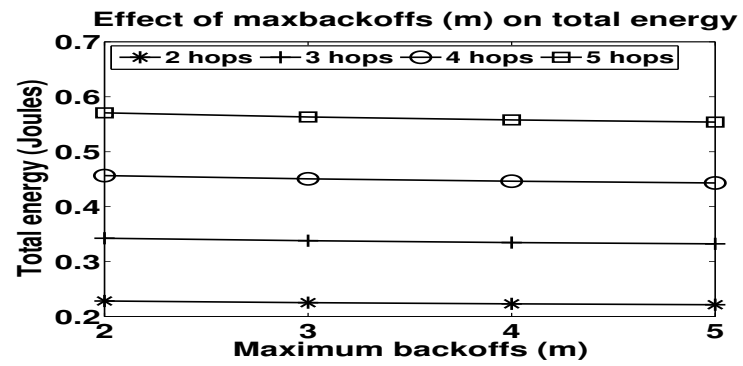

Fig. 6: Effect of maxbackoff stages $(m)$ on Total energy

each curve in Fig. 7 is variation in number of hops from 2 to 5. Total energy is increasing exponentially with increase in $L_{p}$ shown in Fig. 7. Increase in energy is due to increase in transmit, receive and collision retries energy consumption of nodes. Increase in packet length results in more congestion in the network. Total energy increases with increase in number of hops as the number of nodes that contend for the channel increases with large packet size. Total energy is in the range of [0.44 0.65] for 5 hops scenario. Total energy of the multi-hop network increased by $45 \%$ with variation in packet length for 5 hop network. It is observed from the above results that energy model derived in this paper captured the effect of possible MAC and network parameters effectively.

\section{CONCLUSION}

Energy efficiency is utmost importance because of exponential increase in wireless network node density in evolving applications like IoT. Analytical energy model for anycast multihop clustered network is developed using a 3-dimensional Markov chain to transform existing asynchronous wireless networks into Green wireless networks. Each relay node in a

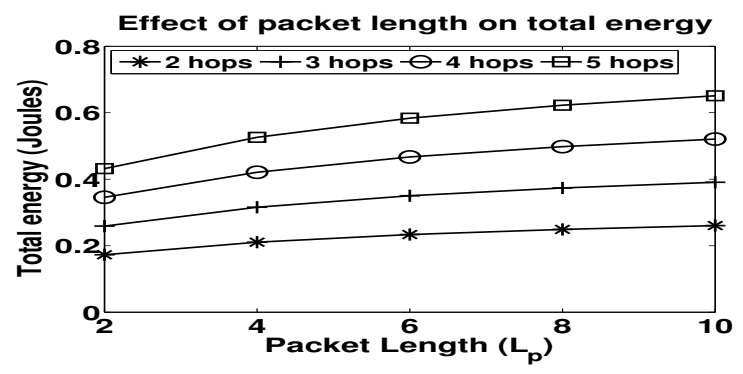

Fig. 7: Effect of packet length $\left(L_{p}\right)$ on Total energy cluster is assigned a poisson wake up rate instead of ideally listening thereby optimizing energy usage of a relay node. Relay node in the model follows anycast multi-hop model removing the dependency on routing layer. Total energy consumption is derived from the proposed model with consideration of MAC parameters and network parameters like $m_{0}, m$, packet length and number of hops. Results show that total energy has higher dependence on minimum backoff exponent $\left(m_{0}\right)$, wake up rate per node $(\mu)$, packet length $\left(L_{p}\right)$ and marginal effect of maximum backoff stages $(m)$ of CSMA/CA. Simultaneous optimization of reliability, delay, energy, MAC and network parameters of the proposed model will result in more accuracy and performance which is the future scope of this paper.

\section{REFERENCES}

[1] IEEE Std 802.15.4-2996, September, Part 15.4: Wireless Medium Access Control (MAC) and Physical Layer (PHY) Specifications for LowRate Wireless Personal Area Networks (WPANs), IEEE, 2006. [Online]. Available: http://www.ieee802.org/15

[2] A. Willig, K. Matheus, and A. Wolisz "Wireless technology in industrial networks", Proceedings of the IEEE, Volume:93, Issue: 6, pp: 1130-1151, June 2005 .

[3] "Smart 2020 report" http://www.smart2020.org/_assets/files/ Smart2020UnitedStatesReportAddendum.pdf

[4] Report by Royal Academy of Engineering "Smart buildings people and performance," can be found at www.raeng.org.uk/societygov/policy/ current $\$ \backslash \$$ issues

[5] S. Pollin, M. Ergen, S. C. Ergen, B. Bougard, L. Van der Perre, I. Moerman, A. Bahai, P. Varaiya, and F. Catthoor,"Performance analysis of slotted carrier sense IEEE 802.15.4 medium access layer," IEEE Transactions in Wireless Communications, vol. 7, no. 9, pp:3359-3371, September 2008.

[6] P. Park, P. Di Marco, C. Fischione, and K. H. Johansson, "Adaptive IEEE 802.15.4 Protocol for Reliable and Timely Communications,", Proceedings of the 9th ACM/IEEE International Conference on Information Processing in Sensor Networks, pp:327-338 2009

[7] C. Fischione, S. Coleri Ergen, P. Park, K. H. Johansson, and A. Sangiovanni- Vincentelli, "Medium Access Control Analytical Modeling and Optimization in Unslotted IEEE 802.15.4 Wireless Sensor Networks,", IIEEE Communications Society Conference on Sensor, Mesh and Ad Hoc Communications and Networks (SECON), pp:1-9, Rome, Italy, June, 2009

[8] P. Park, C. Fischione, A. Bonivento, K. H. Johansson and A. SangiovanniVincentelli, "Breath: an Adaptive Protocol for Industrial Control Applications using Wireless Sensor Networks", IEEE Transactions on Mobile Computing, Volume:10, Issue: 6 June 2011

[9] J. Zheng and M. L. Lee,"A comprehensive performance study of IEEE 802.15.4,", in IEEE Press Book, 2004

[10] A. Koubaa, M. Alves, and E. Tovar, "A comprehensive simulation study of slotted CSMA/CA for IEEE 802.15.4 wireless sensor networks," in IEEE IWFCS, pp:183-192, June 2006,

[11] Zhijia Chen, Chuang Lin,P Hao Wen, Hao Yin "An Analytical Model for Evaluating IEEE 802.15.4 CSMA/CA protocol in Low-rate wireless application", 21st International Conference on Advanced Information Networking and Applications Workshops (AINAW'07) Volume:2 pp:899904, 21-23 May 2007 Niagara Falls

[12] I. RaMAChandran, A. K. Das, and S. Roy, "Analysis of the contention access period of IEEE 802.15.4 MAC," ACM Transactions on Sensor Networks, vol. 3, no. 1, 29 pages, March 2007.

[13] Baobing Wang, Baras, J.S. "Performance Analysis of Time-Critical Peerto-Peer Communications in IEEE 802.15.4 Networks," IEEE ICC 2011 proceedings, pp:1-6, Kyoto 5-9 June 2011

[14] Prasad, Y.R.V, Rajalakshmi P. "Analytical model of adaptive CSMACA MAC for reliable and timely clustered wireless multi-hop communication," IEEE World forum on Internet of Things (WF-IoT) conference, pp:212-217, Seoul 6-8 March 2014

[15] Tmote sky datasheet can be found at www.eecs.harvard.edu/ konrad/ projects/tmote-sky-datasheet 